\title{
Quaderni
}

QUADERNI Communication, technologies, pouvoir

102 | Hiver 2020-2021

Politique(s) des dystopies

\section{Paul Bacot, La métropole de Lyon et les élections métropolitaines}

Cynthia Ghorra-Gobin

\section{OpenEdition}

1 Journals

\section{Édition électronique}

URL : http://journals.openedition.org/quaderni/1917

DOI : 10.4000/quaderni. 1917

ISSN : 2105-2956

Éditeur

Les éditions de la Maison des sciences de l'Homme

\section{Édition imprimée}

Date de publication : 5 janvier 2021

Pagination : 157-160

\section{Référence électronique}

Cynthia Ghorra-Gobin, «Paul Bacot, La métropole de Lyon et les élections métropolitaines », Quaderni [En ligne], 102 | Hiver 2020-2021, mis en ligne le 05 janvier 2021, consulté le 29 janvier 2021. URL : http:// journals.openedition.org/quaderni/1917; DOI : https://doi.org/10.4000/quaderni.1917 


\section{Paul Bacot, \\ La métropole de Lyon et les élections métropolitaines Éditions CRPS-Alain Sitbon, Rillieux-la-Pape, 2019}

\section{Cynthia Ghorra-Gobin}

CNRS-Université Sorbonne Nouvelle Paris 3 
Le deuxième tour des élections municipales qui s'est déroulé en France en juin 2020 s'est traduit par l'arrivée au pouvoir d'élus écologistes dans plusieurs grandes villes, comme Lyon ou Bordeaux. Cette victoire, qui fait suite à la crise sanitaire liée à la pandémie de Covid-19 (perçue comme un prélude aux futures crises liées au changement climatique), a certainement été influencée par le discours d'intellectuels et d'experts militant en faveur d'une politique de transition écologique. À Lyon, la rivalité entre le maire sortant Gérard Collomb (figure nationale) et le candidat écologiste Grégory Doucet (inconnu du grand public), ainsi que les petits arrangements locaux du premier ont fait la une des médias. En raison d'un contexte national et local relativement dense, l'originalité des élections métropolitaines à Lyon (objet du livre de Paul Bacot, professeur à Sciences Po Lyon), a été peu soulignée auprès du grand public.

Dans cet ouvrage qui comble en quelque sorte cette lacune médiatique, il est question de la spécificité des élections métropolitaines à Lyon à venir en 2020, par un spécialiste des élections municipales en France, soulignant le caractère «unique» de la collectivité territoriale de la métropole du Grand Lyon. L'analyse qui en résulte tente d'articuler deux récits : le premier centré sur la politique d'un État centralisé menant une politique de décentralisation, et le second sur le rôle des initiatives locales (complicité entre deux élus, le sénateur-maire de la ville de Lyon et le président du conseil général du Rhône) pour inventer la métropole de Lyon. Dans la partie $2 \mathrm{du}$ premier chapitre, il est dit que la «circonscription départementale» du Rhône (dont la préfecture est Lyon) comprend deux collectivités territoriales : le département du Rhône dont le chef-lieu est Villefranche-sur-Saône et la métropole de Lyon dont le chef-lieu est Lyon. Ce qui signifie que le conseil de la métropole exerce à la fois les compétences départementales et communautaires. L'auteur ajoute aussitôt : «Une telle concentration de compétences décentralisées est unique en France» (p. 14). Il est certes question du local (la métropole de Lyon) mais ce local est aussitôt replacé dans une configuration nationale (État en cours de décentralisation), un positionnement que n'indique pas vraiment le titre.

L'ouvrage qui inclut cartes et tableaux de qualité s'organise en trois parties. La première partie retrace l'histoire de la métropole depuis l'invention de la communauté urbaine en 1969; elle décrit avec précision les différentes instances (conseil, exécutif et autres) ainsi que les relations entre la métropole et les territoires situés dans son environnement immédiat. La deuxième partie relativement technique explique les modalités des élections métropolitaines (modes de scrutin) en 2020 en soulignant l'impératif d'une distinction avec les élections municipales. Le conseil de la métropole se réunit le jeudi qui suit l'élection alors que les conseils municipaux se réunissent le vendredi. La troisième partie intitulée «Lexique métropolitain» définit les termes techniques et juridiques utilisées pour parler de la réalité métropolitaine de Lyon mais certains d'entre eux relèvent tout simplement du répertoire lexical national comme le code général des collectivités territoriales (CGCT), collectivité territoriale, communauté urbaine etc. Elle s'avère extrêmement utile pour toutes les personnes qui ont l'intention de mener un travail de terrain et d'enquêtes sur la métropole de Lyon ou d'en savoir plus sur les mots et définitions indispensables pour décrire le territoire national.

La métropole de Lyon se définit comme une collectivité territoriale de $540 \mathrm{~km}^{2}$ (regroupant 59 communes) qui a hérité des compétences de la communauté urbaine de Lyon et de celles exercées jusqu'avant loi Maptam (2014) par le conseil général du Rhône. En d'autres termes, une loi nationale aura autorisé l'amputation d'une partie du territoire du département du Rhône au profit de la métropole de Lyon : un «fait inédit » dans l'histoire du territoire national écrit l'auteur. Aussi contrairement aux autres métropoles françaises comme Bordeaux, Aix-Marseille, Lille ou Strasbourg pour ne citer que quelquesunes, celle de Lyon n'est pas un EPCI (établis- 
sement public de coopération intercommunale), elle est une collectivité territoriale et à ce titre les membres (150) de son conseil sont élus au suffrage universel par les citoyens des 14 circonscriptions électorales qui la composent. Le conseil élit son président. Le rôle de la métropole est de former un "espace de solidarité pour élaborer et conduire un projet d'aménagement et de développement économique, écologique, social, éducatif, sportif et culturel de son territoire afin d'en améliorer la compétitivité et la cohésion" (Code général des collectivités territoriales). La liste des commissions de la métropole donne une idée de la diversité des domaines de compétence du conseil de la métropole: l'enfance et la famille; les solidarités; l'habitat et le logement; les déplacements (mobilité); eau et assainissement ; propreté; aménagement urbain et grands projets; énergie et environnement; emplois et développement économique; savoirs et culture; attractivité et rayonnement.

Les autres instances de la métropole sont le conseil de développement, la conférence métropolitaine et les conférences territoriales des maires. Le conseil de développement qui rassemble des représentants de milieux économiques, sociaux, éducatifs et culturels a un rôle purement consultatif. Il est organisé en six collèges regroupant deux cents membres. La conférence métropolitaine est également une instance consultative: elle réunit l'ensemble des maires de communes et est présidée par le président de la métropole. Elle élabore ainsi un projet de "pacte de cohérence métropolitain» entre la métropole et les communes. À partir de 2020, cette instance revêtira une importance particulière en raison du mode de scrutin du conseil de la métropole qui ne permet plus aux maires d'en être automatiquement membres.

Le/a lecteur/trice intéressé/e par la métropole de Lyon appréciera la distinction que fait la troisième partie entre l'«aire urbaine " de Lyon, l'«aire métropolitaine lyonnaise » (AML) et la "métropole de Lyon". L'aire urbaine correspond à la définition de l'INSEE qui inclut cinq cents communes avec et autour de la métropole et qui en 2017 compte 2,3 millions d'habitants alors que la métropole n'en compte que 1,4 millions d'habitants. L'AML n'a pas de définition stabilisée, elle correspond aux territoires qui constituent l'Inter-SCoT, instance coordonnant treize syndicats mixtes porteurs de schémas de cohérence territoriale (Scots). Ces trois termes délimitant trois périmètres différents s'avèrent indispensables pour cerner la complexité de la vie politique locale s'inscrivant au-delà d'un régime politique relevant uniquement d'une affiliation nationale. On notera toujours dans cette troisième partie l'appellation Vélo'v qui fait référence au système de bicyclettes en libre-service mis en place en 2005 par la communauté urbaine et qui aura inspiré la création de Vélib à Paris.

Au final, l'ouvrage de Paul Bacot se lit aisément en raison de la clarté de l'écriture et de l'analyse. Il donne à voir une réalité métropolitaine inédite en France et qui pour certains observateurs représenterait le modèle vers lequel devrait tendre les autres métropoles constituées pour le moment en EPCI. Il apporte également un nouvel éclairage sur les enjeux que représente la métropole pour les nouveaux élus. On peut toutefois regretter la préférence qu'accorde l'auteur pour le récit national au détriment de la vie politique locale qui est très riche et qui aura en quelque sorte permis à la loi Maptam de faire de la métropole de Lyon une réalité inédite. Pour de nombreux historiens [Renaud Payre (dir.), 2013, Lyon, ville internationale: la métropole lyonnaise à l'assaut de la scène internationale, Lyon: Libel], Lyon n'est pas la première métropole du pays après Paris, c'est une ville qui au travers de sa bourgeoisie libérale et de politiques ambitieux aura œuvré tout au long du $\mathrm{XX}^{\mathrm{e}}$ siècle pour se doter d'un profil international et qui en ce début de $\mathrm{XXI}^{\mathrm{e}}$ siècle rayonne au-delà de l'horizon national en tant que métropole européenne. En dépit d'une information limitée sur la richesse et la diversité de la vie politique locale - la complicité entre Michel Mercier, le président du conseil général du Rhône et le sénateur-maire de Lyon, Gérard Collomb, en 2012 pour 
influencer la décision de l'État -, cet ouvrage s'avère utile à tout étudiant/e en science politique ou en géographie pour comprendre les progressives transformations structurelles opérées dans la gouvernance des métropoles en France. 